\title{
Self-energy and critical temperature of weakly interacting bosons
}

\author{
Sascha Ledowski, Nils Hasselmann, and Peter Kopietz \\ Institut für Theoretische Physik, Universität Frankfurt, \\ Robert-Mayer-Strasse 8, 60054 Frankfurt, Germany
}

(Dated: November 3, 2003)

\begin{abstract}
Using the exact renormalization group we calculate the momentum-dependent self-energy $\Sigma(k)$ at zero frequency of weakly interacting bosons at the critical temperature $T_{c}$ of Bose-Einstein condensation in dimensions $3 \leq D<4$. We obtain the complete crossover function interpolating between the critical regime $k \ll k_{c}$, where $\Sigma(k) \propto k^{2-\eta}$, and the short-wavelength regime $k \gg k_{c}$, where $\Sigma(k) \propto k^{2(D-3)}$ in $D>3$ and $\Sigma(k) \propto \ln \left(k / k_{c}\right)$ in $D=3$. Our approach yields the crossover scale $k_{c}$ on the same footing with a reasonable estimate for the critical exponent $\eta$ in $D=3$. From our $\Sigma(k)$ we find for the interaction-induced shift of $T_{c}$ in three dimensions $\Delta T_{c} / T_{c} \approx 1.23 a n^{1 / 3}$, where $a$ is the s-wave scattering length and $n$ is the density.
\end{abstract}

PACS numbers: 03.75.Hh, 05.30.Jp, 05.70.Jk

Introduction. Triggered by Nobel Prize winning experiments, the past few years have seen an upsurge of activity in the field of Bose-Einstein condensation [1]. The interacting Bose gas falls into the universality class of the classical $O(2)$ model. While for dimensions $D$ slightly below the upper critical dimension $D=4$ the small parameter $\epsilon=4-D$ can be used to study the critical behavior via controlled perturbative renormalization group (RG) calculations 2], no such small parameter exists in the physically relevant case $D=3$. The problem of BoseEinstein condensation in a three-dimensional Bose gas therefore requires non-perturbative methods.

One of the fundamental properties of the system is the energy dispersion $\epsilon_{k}$ of the elementary excitations. In the vicinity of the critical temperature $T_{c}$, where the correlation length becomes large, interactions qualitatively modify the energy dispersion at long wavelengths. Precisely at $T_{c}$ it is well known that $\epsilon_{k} \propto k^{2-\eta}$ for sufficiently small momenta $k$. In $D=3$ high order re-summations of the $\epsilon$-expansion [3] and recent Monte-Carlo simulations [4] yield the critical exponent $\eta \approx 0.038$. On the other hand, when $k$ exceeds some interaction-dependent crossover scale $k_{c}$, self-energy corrections to the noninteracting dispersion $\rho_{0} k^{2}$ become negligible. Here $\rho_{0}=$ $\hbar^{2} / 2 m$, with some effective mass $m$. Let us describe this crossover in terms of a dimensionless self-energy function

$$
\sigma(x)=\left(\rho_{0} k_{c}^{2}\right)^{-1}\left[\Sigma\left(k_{c} x\right)-\Sigma(0)\right],
$$

where $\Sigma(k)$ is the exact self-energy of the interacting Bose gas at zero frequency. By construction, the crossover occurs at $x=O(1)$. Note that the field-theoretical RG effectively sets $k_{c} \rightarrow \infty$, so that neither $k_{c}$ nor $\sigma(x)$ for $x \gg 1$ can be obtained within that framework.

Naively one might expect that in the short-wavelength regime $x \gg 1$ the scaling function can be calculated perturbatively. Unfortunately, in $D=3$ one encounters infrared divergences even in this regime. Baym et al. 5] recently employed a simple self-consistent re-summation which cures the divergences in $D=3$. However, their result $\eta=1 / 2$ is far from the above mentioned accepted value [3, 4]. Clearly, it would be very useful to have a quantitatively accurate formula for the momentumdependence of the self-energy at $T_{c}$, which interpolates between the critical and the short-wavelength regime. As emphasized in Ref. [5], the shift in the critical temperature $\Delta T_{c}$ due to interactions is closely related to the behavior of the scaling function $\sigma(x)$; at weak coupling and to leading order in the scattering length, $\Delta T_{c}$ in $D=3$ is proportional to the crossover scale $k_{c}$, with a constant of proportionality that depends on the specific form of $\sigma(x)$ for $0 \leq x \leq \infty$.

Exact $R G$ flow equations for interacting bosons. Previously Bijlsma and Stoof [6] used RG methods to investigate the infrared behavior of the weakly interacting Bose gas above and below $T_{c}$. However, these authors considered only the constant parts of the vertices. In particular, no attempt was made to calculate the momentumdependence of the self-energy. Here we study this problem using the exact RG in the form proposed by Wetterich 7], and by Morris [8]. In this approach one considers the RG flow of the generating functional of the one-particle irreducible vertices $\Gamma_{\Lambda}^{(2 n)}$ as an infrared cutoff $\Lambda$ is reduced. Assuming that the bosons are in the normal state [9], the exact flow equation for the irreducible two-point vertex is 10, 11]

$$
\partial_{\Lambda} \Gamma_{\Lambda}^{(2)}(K)=\int_{K^{\prime}} \dot{G}_{\Lambda}\left(K^{\prime}\right) \Gamma_{\Lambda}^{(4)}\left(K, K^{\prime} ; K^{\prime}, K\right),
$$

where for sharp infrared cutoff $\Lambda$

$$
\dot{G}_{\Lambda}(K)=\frac{\delta(|\mathbf{k}|-\Lambda)}{i \omega_{n}-\epsilon_{\mathbf{k}}+\mu-\Sigma(0, i 0)-\Gamma_{\Lambda}^{(2)}(K)} .
$$

We use the notation $K=\left(\mathbf{k}, i \omega_{n}\right), \int_{K}=(\beta V)^{-1} \sum_{\mathbf{k}, \omega_{n}}$, where $\beta$ is the inverse temperature, $V$ is the volume, and $\omega_{n}=2 \pi n T$ are bosonic Matsubara frequencies. The irreducible four-point vertex satisfies 


$$
\begin{aligned}
& \partial_{\Lambda} \Gamma_{\Lambda}^{(4)}\left(K_{1}^{\prime}, K_{2}^{\prime} ; K_{2}, K_{1}\right)=\int_{K} \dot{G}_{\Lambda}(K) \Gamma_{\Lambda}^{(6)}\left(K_{1}^{\prime}, K_{2}^{\prime}, K ; K, K_{2}, K_{1}\right) \\
& \quad+\int_{K}\left[\dot{G}_{\Lambda}(K) G_{\Lambda}\left(K^{\prime}\right) \Gamma_{\Lambda}^{(4)}\left(K_{1}^{\prime}, K_{2}^{\prime} ; K^{\prime}, K\right) \Gamma_{\Lambda}^{(4)}\left(K, K^{\prime}, K_{2}, K_{1}\right)\right]_{K^{\prime}=K_{1}+K_{2}-K} \\
& \quad+\int_{K}\left[\left[\dot{G}_{\Lambda}(K) G_{\Lambda}\left(K^{\prime}\right)+G_{\Lambda}(K) \dot{G}_{\Lambda}\left(K^{\prime}\right)\right] \Gamma_{\Lambda}^{(4)}\left(K_{1}^{\prime}, K^{\prime} ; K, K_{1}\right) \Gamma_{\Lambda}^{(4)}\left(K_{2}^{\prime}, K ; K^{\prime}, K_{2}\right)\right]_{K^{\prime}=K_{1}-K_{1}^{\prime}+K} \\
& \quad+\int_{K}\left[\left[\dot{G}_{\Lambda}(K) G_{\Lambda}\left(K^{\prime}\right)+G_{\Lambda}(K) \dot{G}_{\Lambda}\left(K^{\prime}\right)\right] \Gamma_{\Lambda}^{(4)}\left(K_{2}^{\prime}, K^{\prime} ; K, K_{1}\right) \Gamma_{\Lambda}^{(4)}\left(K_{1}^{\prime}, K ; K^{\prime}, K_{2}\right)\right]_{K^{\prime}=K_{1}-K_{2}^{\prime}+K} .
\end{aligned}
$$

Here the exact propagator $G_{\Lambda}(K)$ is obtained from Eq. (31) by replacing $\delta(|\mathbf{k}|-\Lambda) \rightarrow \Theta(|\mathbf{k}|-\Lambda)$. The flow equation for the irreducible six-point vertex $\Gamma_{\Lambda}^{(6)}$ on the righthand side of Eq. (4) can be found in Ref. [10]. Because quantum mechanics is irrelevant for classical critical phenomena, for our purpose it is sufficient to retain only the zero frequency part of all vertices and work with an effective classical theory, with ultraviolet cutoff given by $\Lambda_{0}=2 \pi / \lambda_{\text {th }}$, where $\lambda_{\text {th }}=h / \sqrt{2 \pi m T}$ is the thermal de Broglie wavelength. In principle, the above flow equations can also be used to calculate the parameters of the effective classical model, which are renormalized by the degrees of freedom involving non zero Matsubara frequencies. Here we take these finite renormalizations implicitly into account via the initial conditions at scale $\Lambda_{0}$. In particular, in $D=3$ we write the initial value of the four-point vertex as $\Gamma_{\Lambda_{0}}^{(4)}(0,0 ; 0,0)=16 \pi \rho_{0} a$, where $a$ is the $s$-wave scattering length.

To study the critical regime, it is convenient to introduce dimensionless momenta $\mathbf{q}=\mathbf{k} / \Lambda$ and the rescaled classical propagator $\tilde{G}_{l}(q)=-\left(\rho_{0} \Lambda^{2} / Z_{l}\right) G_{\Lambda}(\mathbf{q} \Lambda, i 0)=$ $\Theta(q-1) / R_{l}(q)$, which is a function of the logarithmic flow parameter $l=-\ln \left(\Lambda / \Lambda_{0}\right)$. Here $R_{l}(q)=Z_{l} q^{2}+\tilde{\Gamma}_{l}^{(2)}(q)$, where $\tilde{\Gamma}_{l}^{(2)}(q)=Z_{l}\left(\rho_{0} \Lambda^{2}\right)^{-1} \Gamma_{\Lambda}^{(2)}(\Lambda \mathbf{q}, i 0)$ is the rescaled two-point vertex, and $Z_{l}=1-\partial \tilde{\Gamma}_{l}^{(2)}(q) /\left.\partial q^{2}\right|_{q^{2}=0}$ is the wave-function renormalization factor. For $n \geq 2$ we define the rescaled higher-order vertices

$$
\begin{aligned}
\tilde{\Gamma}_{l}^{(2 n)}\left(\mathbf{q}_{i}\right) & =\left(K_{D} T\right)^{n-1} \Lambda^{D(n-1)-2 n}\left(Z_{l} / \rho_{0}\right)^{n} \\
& \times \Gamma_{\Lambda}^{(2 n)}\left(\mathbf{k}_{i} \rightarrow \Lambda \mathbf{q}_{i}, \omega_{n_{i}} \rightarrow 0\right) .
\end{aligned}
$$

For later convenience we have inserted the numerical factor $K_{D}=\Omega_{D} /(2 \pi)^{D}$, where $\Omega_{D}$ is the surface area of the $D$-dimensional unit sphere. The rescaled vertices satisfy exact flow equations of the form

$$
\begin{aligned}
& \partial_{l} \tilde{\Gamma}_{l}^{(2 n)}\left(\mathbf{q}_{i}\right)=\left[2 n-D(n-1)-n \eta_{l}\right. \\
& \left.-\sum_{i=1}^{n}\left(\mathbf{q}_{i}^{\prime} \cdot \nabla_{\mathbf{q}_{i}^{\prime}}+\mathbf{q}_{i} \cdot \nabla_{\mathbf{q}_{i}}\right)\right] \tilde{\Gamma}_{l}^{(2 n)}\left(\mathbf{q}_{i}\right)+\dot{\Gamma}_{l}^{(2 n)}\left(\mathbf{q}_{i}\right),
\end{aligned}
$$

where $\eta_{l}=\partial \dot{\Gamma}_{l}^{(2)}(q) /\left.\partial q^{2}\right|_{q=0}$ is the flowing anomalous dimension. The function $\dot{\Gamma}_{l}^{(2)}(q)$ on the right-hand side of the flow function of the two-point vertex is

$$
\dot{\Gamma}_{l}^{(2)}(q)=\int_{\mathbf{q}^{\prime}} \dot{G}_{l}\left(q^{\prime}\right) \tilde{\Gamma}_{l}^{(4)}\left(\mathbf{q}, \mathbf{q}^{\prime} ; \mathbf{q}^{\prime}, \mathbf{q}\right)
$$

with $\int_{\mathbf{q}}=\int d^{D} q / \Omega_{D}$ and $\dot{G}_{l}(q)=\delta(q-1) / R_{l}(q)$. The flow function $\dot{\Gamma}_{l}^{(4)}\left(\mathbf{q}_{1}^{\prime}, \mathbf{q}_{2}^{\prime} ; \mathbf{q}_{2}, \mathbf{q}_{1}\right)$ can be obtained from the right-hand side of Eq. (4) by replacing $\dot{G}_{\Lambda}(K) \rightarrow$ $-\dot{G}_{l}(q), G_{\Lambda}(K) \rightarrow-\tilde{G}_{l}(q), \Gamma_{\Lambda}^{(2 n)} \rightarrow \tilde{\Gamma}_{l}^{(2 n)}, \int_{K} \rightarrow \int_{\mathbf{q}}$, and multiplying the resulting expression by an overall minus sign. From the above definitions we can show [12] that at $T_{c}$ the function (1) can be written as

$$
\sigma(x)=\int_{0}^{\infty} d l e^{-2\left(l-l_{c}\right)+\int_{0}^{l} d \tau \eta_{\tau}} \dot{\Gamma}_{l}^{(2-r)}\left(e^{l-l_{c}} x\right),
$$

where $\dot{\Gamma}_{l}^{(2-r)}(q)=\dot{\Gamma}_{l}^{(2)}(q)-\dot{\Gamma}_{l}^{(2)}(0)$ and $l_{c}$ will be determined shortly. Hence, the flow function (7) is the key quantity for obtaining $\sigma(x)$ via the RG.

Classification of couplings and crossover scale. In $3 \leq$ $D<4$ there are two relevant couplings: the momentumindependent parts $r_{l}=\tilde{\Gamma}_{l}^{(2)}(0)$ and $u_{l}=\tilde{\Gamma}_{l}^{(4)}(0,0 ; 0,0)$ of the two-point and four-point vertices. If we simply ignore all other couplings, the flow of $r_{l}$ and $u_{l}$ is determined by

$$
\begin{aligned}
& \partial_{l} r_{l}=\left(2-\eta_{l}\right) r_{l}+u_{l} /\left(1+r_{l}\right), \\
& \partial_{l} u_{l}=\left(\epsilon-2 \eta_{l}\right) u_{l}-\frac{5}{2} u_{l}^{2} /\left(1+r_{l}\right)^{2} .
\end{aligned}
$$

Note that the initial value $r_{0}$ has to be fine tuned so that $r_{*}=\lim _{l \rightarrow \infty} r_{l}$ is finite, corresponding to the critical point. As long as $\left|r_{l}\right| \ll 1$ and $\eta_{l} \ll \epsilon$, the qualitative behavior of the solution of Eq. (10) can be obtained by simply replacing $r_{l} \rightarrow 0$ and $\eta_{l} \rightarrow 0$ on the righthand side. Then the solution of Eq. (10) is a Fermi function, $u_{l} / u_{*}=\left[e^{\epsilon\left(l_{c}-l\right)}+1\right]^{-1}$, where $u_{*}=2 \epsilon / 5$ and $l_{c}=\epsilon^{-1} \ln \left(u_{*} / u_{0}-1\right) \approx \epsilon^{-1} \ln \left(u_{*} / u_{0}\right)$. Throughout this work we assume that $u_{0} \ll u_{*}$, corresponding to weak bare interactions. Thus, within a narrow interval of width $1 / \epsilon$ centered at $l=l_{c}$ the coupling $u_{l}$ suddenly approaches its fixed point value $u_{*}$. Below we shall show that $k_{c}=\Lambda_{0} e^{-l_{c}} \approx \Lambda_{0}\left(u_{0} / u_{*}\right)^{1 / \epsilon}$ is the scale where the dispersion crosses over from $k^{2}$ to the $k^{2-\eta}$. 
We now discuss the marginal couplings. For $3<D<4$ the only marginal parameter is the wave-function renormalization $Z_{l}$; it satisfies by construction $\partial_{l} Z_{l}=-\eta_{l} Z_{l}$. In $D=3$, there are three additional marginal couplings. The most obvious one [ 6$]$ is the momentum-independent part of the six-point vertex, $v_{l}=\tilde{\Gamma}_{l}^{(6)}(0,0,0 ; 0,0,0)$. But there are two more marginal couplings $a_{l}$ and $b_{l}$ related to the four-point vertex, which has the expansion for small momenta

$$
\begin{aligned}
\tilde{\Gamma}_{l}^{(4)}\left(\mathbf{q}_{1}^{\prime}, \mathbf{q}_{2}^{\prime} ; \mathbf{q}_{2}, \mathbf{q}_{1}\right) & =u_{l}+a_{l}\left(\left|\mathbf{q}_{1}-\mathbf{q}_{1}^{\prime}\right|+\left|\mathbf{q}_{1}-\mathbf{q}_{2}^{\prime}\right|\right) \\
& +b_{l}\left|\mathbf{q}_{1}+\mathbf{q}_{2}\right|+O\left(\mathbf{q}_{i}^{2}\right) .
\end{aligned}
$$

One easily verifies that in $D=3$ the scaling dimensions of $a_{l}$ and $b_{l}$ vanish. Note that even if initially $\tilde{\Gamma}_{l=0}^{(4)}=u_{0}$ is momentum-independent (corresponding to the initial conditions $a_{0}=b_{0}=0$ ), finite values of $a_{l}$ and $b_{l}$ are generated as we iterate the RG.

Truncation and anomalous dimension. To calculate $\eta_{l}$, we need the momentum-dependent part of the fourpoint vertex. Note that in $D>3$ the momentumdependence of $\tilde{\Gamma}_{l}^{(4)}\left(\mathbf{q}_{1}^{\prime}, \mathbf{q}_{2}^{\prime} ; \mathbf{q}_{2}, \mathbf{q}_{1}\right)$ is irrelevant. As explained in Refs. [13, 14], for sufficiently large $l$ irrelevant couplings become local functions of the relevant and marginal ones. Assuming $u_{l} \ll 1$, the momentumdependence of $\tilde{\Gamma}_{l}^{(4)}\left(\mathbf{q}_{1}^{\prime}, \mathbf{q}_{2}^{\prime} ; \mathbf{q}_{2}, \mathbf{q}_{1}\right)$ can be obtained by setting $\tilde{\Gamma}_{l}^{(6)} \rightarrow 0$ and $\tilde{\Gamma}_{l}^{(4)} \rightarrow u_{l}$ in the expression for the flow function $\dot{\Gamma}_{l}^{(4)}\left(\mathbf{q}_{1}^{\prime}, \mathbf{q}_{2}^{\prime} ; \mathbf{q}_{2}, \mathbf{q}_{1}\right)$. In Ref. 15] we have used a similar truncation to calculate the spectral function of the Tomonaga-Luttinger model using the exact RG. In the present problem this truncation amounts to

$$
\begin{array}{r}
\dot{\Gamma}_{l}^{(4)}\left(\mathbf{q}_{1}^{\prime}, \mathbf{q}_{2}^{\prime} ; \mathbf{q}_{2}, \mathbf{q}_{1}\right) \approx-u_{l}^{2}\left[\frac{1}{2} \dot{\chi}_{l}\left(\left|\mathbf{q}_{1}+\mathbf{q}_{2}\right|\right)\right. \\
\left.+\dot{\chi}_{l}\left(\left|\mathbf{q}_{1}-\mathbf{q}_{1}^{\prime}\right|\right)+\dot{\chi}_{l}\left(\left|\mathbf{q}_{1}-\mathbf{q}_{2}^{\prime}\right|\right)\right],
\end{array}
$$

where the generalized susceptibility is given by $\dot{\chi}_{l}(q)=$ $2 \int_{\mathbf{q}^{\prime}} \dot{G}_{l}\left(\left|\mathbf{q}^{\prime}\right|\right) \tilde{G}_{l}\left(\left|\mathbf{q}^{\prime}+\mathbf{q}\right|\right)$. The approximation (12) can be formally justified as long as $u_{l}$ remains small, which is certainly the case for small $\epsilon$. Unfortunately, in $D=3$ the renormalized $u_{*}$ is not guaranteed to be small. Moreover, the couplings $v_{l}, a_{l}$ and $b_{l}$ which become marginal in $D=3$ are not consistently taken into account in Eq. (12), so that a priori we cannot estimate the accuracy of our truncation in $D=3$. Nevertheless, we shall use Eq. (12) as a first approximation even in $D=3$; an improved truncation including the marginal couplings $v_{l}, a_{l}$ and $b_{l}$ will be presented elsewhere 12].

Given the truncation (12), the exact flow equation (6) for $\tilde{\Gamma}_{l}^{(4)}\left(\mathbf{q}_{1}^{\prime}, \mathbf{q}_{2}^{\prime} ; \mathbf{q}_{2}, \mathbf{q}_{1}\right)$ can be solved without further approximation. Substituting the result into Eq. (7) we obtain for the function $\dot{\Gamma}_{l}^{(2-r)}(q)$ required in Eq. (8)

$$
\begin{gathered}
\dot{\Gamma}_{l}^{(2-r)}(q) \approx-\frac{3}{2\left(1+r_{l}\right)} \int_{0}^{l} d t e^{\epsilon t-2 \int_{l-t}^{l} d \tau \eta_{\tau}} u_{l-t}^{2} \\
\times\left\langle\dot{\chi}_{l-t}\left(e^{-t}\left|\hat{\mathbf{q}}^{\prime}+\mathbf{q}\right|\right)-\dot{\chi}_{l-t}\left(e^{-t}\right)\right\rangle_{\hat{\mathbf{q}}^{\prime}},
\end{gathered}
$$

where $\langle\ldots\rangle_{\hat{\mathbf{q}}^{\prime}}$ denotes angular average over the unit vector $\hat{\mathbf{q}}^{\prime}$. Eq. (13) implies the following integral equation for the flowing anomalous dimension $\eta_{l}=\partial \dot{\Gamma}_{l}^{(2)}(q) /\left.\partial q^{2}\right|_{q=0}$,

$$
\eta_{l}=\int_{0}^{l} d t K(l, t) u_{l-t}^{2} e^{-2 \int_{l-t}^{l} d \tau \eta_{\tau}},
$$

with the kernel given by

$$
\begin{gathered}
K(l, t)=-\frac{3}{4 D\left(1+r_{l}\right)}\left[(D-1) e^{-(D-3) t} \dot{\chi}_{l-t}^{\prime}\left(e^{-t}\right)\right. \\
\left.+e^{-(D-2) t} \dot{\chi}_{l-t}^{\prime \prime}\left(e^{-t}\right)\right]
\end{gathered}
$$

where $\dot{\chi}_{l}^{\prime}(x)=d \dot{\chi}_{l}(x) / d x$ and $\dot{\chi}_{l}^{\prime \prime}(x)=d^{2} \dot{\chi}_{l}(x) / d x^{2}$. Eq. (14) together with the two flow equations (910) form a system of three coupled integro-differential equations for the three unknown functions $r_{l}, u_{l}$ and $\eta_{l}$. For small $\epsilon$ we recover the known result $\eta=\lim _{l \rightarrow \infty} \eta_{l}=\epsilon^{2} / 50$. In $D=3$ we find numerically at the fixed point $r_{*}=-0.143$, $u_{*}=0.232$, and $\eta=0.104$, which should be compared with the accepted value $\eta=0.038$ 2, 3, 4]. Given the simplicity of our truncation, it is quite satisfactory that our estimate for $\eta$ has the correct order of magnitude. We can substantially improve our estimate for $\eta$ by including the above mentioned marginal couplings [16]. In comparison, the method of Ref. [5] yields $\eta=0.5$.

Scaling function. To obtain the scaling function $\sigma(x)$ we substitute our approximation (13) into Eq. (8). The integrations can be simplified by noting that the flow of $\eta_{\tau}$ on the right-hand side can be approximated by a Fermi function, $\eta_{\tau}=\eta\left[e^{\epsilon\left(l_{c}-\tau\right)}+1\right]^{-1}$. We have verified numerically that an analogous substitution in Eq. (14) yields a good approximation to the true solution $\eta_{l}$. Introducing appropriate integration variables we obtain

$$
\sigma(x)=\frac{3 u_{*}^{2}}{2} x^{2-\eta} \int_{x e^{-l_{c}}}^{\infty} d y \frac{y^{-3+2 \epsilon} F\left(x, y ; \eta, l_{c}\right)}{\left[x^{\epsilon}+y^{\epsilon}\right]^{2-2 \eta / \epsilon}},
$$

with the dimensionless function

$$
\begin{aligned}
& F\left(x, y ; \eta, l_{c}\right)=\int_{0}^{1} d z \frac{z^{1-\epsilon}}{\left[x^{\epsilon}+(y / z)^{\epsilon}\right]^{\eta / \epsilon}\left[1+r_{l_{c}+\ln \frac{y}{z x}}\right]} \\
& \times\left\langle\dot{\chi}_{l_{c}+\ln \frac{y}{x}}(z)-\dot{\chi}_{l_{c}+\ln \frac{y}{x}}\left(\left|z \hat{\mathbf{q}}^{\prime}+y \hat{\mathbf{q}}\right|\right)\right\rangle_{\hat{\mathbf{q}}^{\prime}} .
\end{aligned}
$$

A numerical evaluation of Eq. (16) is shown in Fig. 10 The asymptotic behavior for small and large $x$ can be extracted analytically. In the critical regime $x \ll 1$ we find in $D$ dimensions $\sigma(x) \sim A_{D} x^{2-\eta}$, with $A_{D}=$ $\frac{3}{2} u_{*}^{2} \int_{0}^{\infty} d y y^{-3+2 \eta} F(0, y ; \eta, \infty)$. In $D=3$ we obtain numerically $A_{3}=1.20$. On the other hand, in the short wavelength regime we find $\sigma(x) \sim B_{D} x^{2(D-3)}$ for $[2(D-3)]^{-1} \ll \ln x$, and $\sigma(x) \sim B_{3}^{\prime} \ln x$ for $1 \ll \ln x \ll$ $[2(D-3)]^{-1}$ (which includes the limit $D \rightarrow 3$ ). Here $B_{D} \propto u_{*}^{2} /(D-3)$ and $B_{3}^{\prime}=3 \pi^{2} u_{*}^{2} / 24$. Keeping in mind that $k_{c} \propto\left(u_{0} / u_{*}\right)^{1 / \epsilon}$, it is easy to see that for $k \gg k_{c}$ the physical self-energy $\Sigma(k)-\Sigma(0)$ is proportional to $u_{0}^{2}$. 


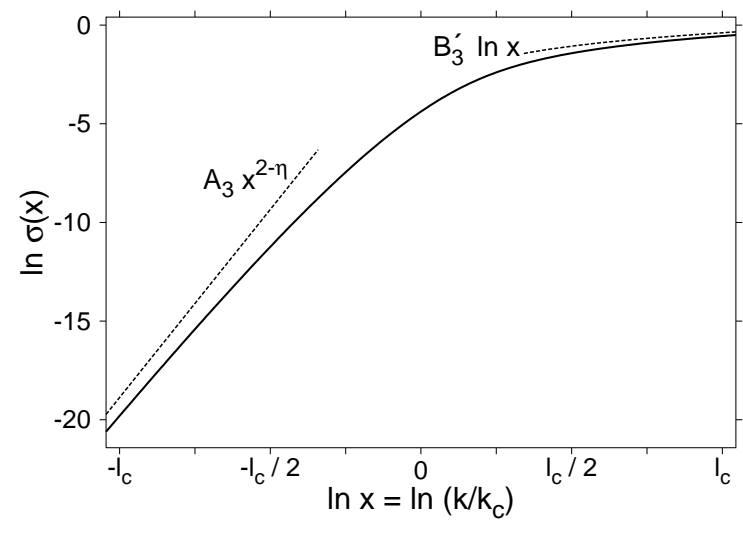

FIG. 1: Double logarithmic plot of the scaling function $\sigma(x)$ in $D=3$, see Eqs. (1) and (16). Note that in our rescaled units we obtain a universal curve for $u_{0} \rightarrow 0$. The dashed lines indicate the asymptotic behavior for small and large $x$.

Critical temperature. Given $\sigma(x)$, we may calculate the interaction-induced shift $\Delta T_{c}$ of the critical temperature of the weakly interacting Bose gas. Generalizing an expression given by Baym et al. [5] for arbitrary $D$, we obtain for the contribution from classical fluctuations

$$
\frac{\Delta T_{c}}{T_{c}}=\frac{2 \Omega_{D}\left(u_{0} / u_{*}\right)^{\frac{D-2}{4-D}}}{\pi D \zeta(D / 2)} \int_{0}^{\Lambda_{0} / k_{c}} d x \frac{x^{D-3} \sigma(x)}{x^{2}+\sigma(x)} .
$$

Keeping in mind that $\sigma(x) \sim B_{D} x^{2(D-3)}$ for $D>3$ and large $x$, it is easy to see that we may remove the ultraviolet cutoff $\left(\Lambda_{0} \rightarrow \infty\right)$ in Eq. (18) provided $\frac{D-2}{4-D}<2$, i.e. $D<10 / 3$. Only in this case $\Delta T_{c}$ is dominated by classical fluctuations. For $D \geq 10 / 3$ the value of the integral in Eq. (18) depends on the ultraviolet cutoff, such that it is proportional to $u_{0}^{2}$, with logarithmic corrections $\left(\propto u_{0}^{2} \ln u_{0}\right)$ in $D=10 / 3$. In three dimensions, where $u_{0}=16 \pi^{-1}[\zeta(3 / 2)]^{-1 / 3} a n^{1 / 3}$, we find [17] to leading order $\Delta T_{c} \approx c_{1} a n^{1 / 3} T_{c}$ with $c_{1}=1.23$, in agreement with the five-loop result $c_{1}=1.14 \pm 0.11$ by Kleinert 18 and the seven-loop result $c_{1}=1.27 \pm 0.11$ by Kastening [19]. For recent reviews on the problem of calculating $\Delta T_{c}$ see Ref. 20 .

Summary and outlook. In this work we have used the exact RG to calculate the momentum-dependent selfenergy of weakly interacting bosons at the critical point. Our central result is an explicit expression for the scaling function $\sigma\left(k / k_{c}\right)$ (see Eqs. (11) and (16)) which interpolates between the critical regime $k \ll k_{c}$ and the shortwavelength regime $k \gg k_{c}$. Note that the momentumdependent part of Eq. (12) contains an infinite set of irrelevant (and hence non-renormalizable) couplings, so that neither the crossover scale $k_{c}$ nor $\Sigma(k)$ for $k \gtrsim k_{c}$ are accessible within field-theoretical RG. The crossover function shown in Fig. 11 should be useful for a comparison with experiments probing the single-particle excitations in a wide range of length scales, for example via mea- surements of the velocity distribution. We have shown here how the exact RG can be employed to calculate full scaling functions and not just their asymptotic behavior. Our method is quite general and should prove useful in the study of other critical systems, for example Luttinger liquids [15] or systems described by the dilute Bose gas quantum critical point 21].

This work was partially supported by the DFG via Forschergruppe FOR 412.

[1] See, for example, L. Pitaevskii and S. Stringari, BoseEinstein Condensation, (Clarendon Press, Oxford, 2003).

[2] J. Zinn-Justin, Quantum Field Theory and Critical Phenomena, (Clarendon Press, Oxford, 3rd edition, 1996).

[3] R. Guida and J. Zinn-Justin, J. Phys. A 31, 8103 (1998).

[4] M. Hasenbusch and T. Török, J. Phys. A 32, 6361 (1999).

[5] G. Baym, J.-P. Blaizot, M. Holzmann, F. Laloe, and D. Vautherin, Phys. Rev. Lett. 83, 1703 (1999); Eur. Phys. J. B 24, 107 (2001).

[6] M. Bijslma and H. T. C. Stoof, Phys. Rev. A 54, 5085 (1996).

[7] C. Wetterich, Phys. Lett. B 301, 90 (1993).

[8] T. R. Morris, Int. J. Mod. Phys. A 9, 2411 (1994).

[9] For a recent RG study of the superfluid phase at $T=0$ see F. Pistolesi, C. Castellani, C. Di Castro, and G. C. Strinati, cond-mat/0310306

[10] P. Kopietz and T. Busche, Phys. Rev. B 64, 155101 (2001).

[11] Following Ref. [10] we define $\Gamma_{\Lambda}^{(2)}(K)=\Sigma_{\Lambda}(K)-\Sigma(0, i 0)$, where $\Sigma_{\Lambda}(K)$ is the self-energy with infrared cutoff $\Lambda$ and $\Sigma(0, i 0)$ is the exact self-energy at vanishing momentum and frequency. At the critical point $\mu=\Sigma(0, i 0)$, so that neither $\mu$ nor $\Sigma(0, i 0)$ explicitly appear in our flow equations. The counter-term $\Sigma(0, i 0)$ can be reconstructed from the flow of the marginal and relevant couplings [10], see also S. Ledowski and P. Kopietz, J. Phys.: Condens. Matter 15, 4779 (2003).

[12] S. Ledowski, N. Hasselmann, and P. Kopietz, unpublished.

[13] J. Polchinski, Nucl. Phys. B 231, 269 (1984).

[14] P. Kopietz, Nucl. Phys. B 595, 493 (2001).

[15] T. Busche, L. Bartosch, and P. Kopietz, J. Phys.: Condens. Matter 14, 8513 (2002).

[16] Retaining apart from the relevant and marginal parameters $r_{l}, u_{l}, v_{l}, a_{l}$, and $b_{l}$ also the irrelevant couplings proportional to $u_{l}^{2}$ contained in Eq. (12), we obtain $\eta=0.034$ in $D=3$ [12], which is quite close to the accepted value $\eta=0.038$.

[17] Since we are interested in the leading term in powers of $a$, we may simplify the integrand in Eq. (17) by approximating $r_{l} \approx r_{*}\left[e^{l_{c}-l}+1\right]^{-1}$, which is quantitatively accurate for $l \gtrsim l_{c}$ and leads to negligible corrections for $l \lesssim l_{c}$. If we simply set $r_{l} \rightarrow 0$ in Eq. (17) we obtain $c_{1} \approx 0.928$, while the replacement $r_{l} \rightarrow r_{*}$ yields $c_{1} \approx 1.52$.

[18] H. Kleinert, cond-mat/0210162 3.

[19] B. Kastening, cond-mat/0309060 cond-mat/0303486

[20] M. Haque, cond-mat/0302076 J. O. Andersen, cond-mat/0305138

[21] See, for example, S. Sachdev, Quantum Phase Transitions, (Cambridge University Press, Cambridge, 2000). 